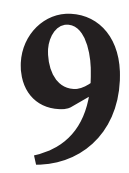

\title{
¿FORMA EN SOSTENIBILIDAD EL SISTEMA UNIVERSITARIO ESPAÑOL? VISIÓN DEL ALUMNADO DE CUATRO UNIVERSIDADES 1
}

\section{(DOES THE SPANISH UNIVERSITY SYSTEM TEACH SUSTAINABILITY? PERCEPTION OF THE STUDENTS OF FOUR UNIVERSITIES)}

Rocío Valderrama-Hernández

Lucía Alcántara Rubio

Universidad de Sevilla

Fermín Sánchez-Carracedo

Universitat Politècnica de Catalunya

David Caballero

Sara Serrate

Universidad de Salamanca

Dolors Gil-Doménech

Salvador Vidal-Raméntol

Universitat Internacional de Catalunya

Rafael Miñano

Universidad Politécnica de Madrid

DOI: $10.5944 / e d u c X X 1.23420$

Cómo referenciar este artículo/How to reference this article:

Valderrama-Hernández, R.; Alcántara Rubio, L.; Sánchez-Carracedo, F.; Caballero, D.; Serrate, S.; Gil-Doménech, D.; Vidal-Raméntol, S., y Miñano, R. (2020). ¿Forma en sostenibilidad el sistema universitario español? Visión del alumnado de cuatro universidades. Educación XX1, 23(1), 221-245, doi: 10.5944/educXX1.23420

Valderrama-Hernández, R.; Alcántara Rubio, L.; Sánchez-Carracedo, F.; Caballero, D.; Serrate, S.; Gil-Doménech, D.; Vidal-Raméntol, S., \& Miñano, R. (2020). Does the Spanish university system teach sustainability? Perception of the students of four universities. Educación XX1, 23(1), 221-245, doi: 10.5944/educXX1.23420

\section{RESUMEN}

Desde hace más de una década, las universidades han puesto en marcha diversas iniciativas internacionales y nacionales para asumir un rol de liderazgo en la promoción de una educación para el desarrollo sostenible, 

¿FORMA EN SOSTENIBILIDAD EL SISTEMA UNIVERSITARIO ESPAÑOL?

y para dar respuestas a los retos globales de la sociedad actual. Es preciso analizar la efectividad de esas iniciativas, y preguntarnos si las universidades están formando a sus estudiantes para afrontar con responsabilidad dichos retos.

Este es uno de los objetivos del proyecto EDINSOST, dentro de cuyo marco hemos llevado a cabo una investigación de tipo cualitativa, desarrollando grupos de discusión con estudiantes de últimos cursos de distintas universidades y perfiles académicos. Esto nos ha permitido integrar en nuestros análisis la perspectiva del alumnado universitario.

En este artículo se presentan las aportaciones más relevantes en relación a la visión que tienen los estudiantes sobre la sostenibilidad, su experiencia formativa al respecto en la universidad y su opinión sobre cómo podría mejorarse esta formación. Los resultados muestran que la formación en sostenibilidad que aporta la universidad es insuficiente, y que los estudiantes no se sienten preparados para integrar la sostenibilidad en su actividad profesional. Sin embargo, consideran fundamental que la universidad incluya la sostenibilidad en sus currículos académicos, y enfatizan la importancia de emplear metodologías activas que conecten al alumnado con la realidad y fomenten la reflexión.

La investigación muestra que queda mucho trabajo por hacer para integrar la sostenibilidad en la formación universitaria. Las propuestas realizadas por alumnado están alineadas con las de los expertos en educación para la sostenibilidad. Para llevarlas a cabo se requiere tanto la formación y compromiso del profesorado como la implicación institucional.

\section{PALABRAS CLAVE}

Sostenibilidad; desarrollo sostenible; grupos de discusión; opinión del alumnado; competencias en sostenibilidad; formación universitaria en sostenibilidad; proyecto EDINSOST.

\section{ABSTRACT}

For more than a decade, various national and international initiatives have emerged so that universities can assume a leadership role in order to promote education for sustainable development and to respond to the global challenges of today's society. It is necessary to analyze the effectiveness of these initiatives, and to ask ourselves whether or not universities are training their students to face these challenges with responsibility. 

¿FORMA EN SOSTENIBILIDAD EL SISTEMA UNIVERSITARIO ESPAÑOL?

This is one of the objectives of the EDINSOST project. Within the framework of EDINSOST, we have carried out qualitative research, developing focus groups with students in their final years at different universities and different academic profiles. This has allowed us to integrate the perspective of university students into our analysis.

In this paper, we present the most relevant contributions in relation to the students' vision of sustainability, their formative experience in the university and their opinion on how it could be improved. Results show that the sustainability training provided by university is insufficient: students do not feel prepared to integrate sustainability into their professional activity. However, they consider it essential that universities should include sustainability in their academic curricula, and they emphasize the importance of using active methodologies that connect students with reality and encourage reflection.

The research shows that there is a lot of work to be done in order to integrate sustainability into university education. Students' proposals are aligned with those of experts in education for sustainability. To carry them out requires both teacher training and commitment, as well as institutional involvement.

\section{KEY WORDS}

Sustainability; sustainable development; focus groups; student opinion; competencies in sustainability; university education in sustainability; EDINSOST project.

\section{INTRODUCCIÓN}

La educación debería tener como eje central la formación de individuos responsables, que participen en una sociedad sostenible (Pérez-Franco, de Pro-Bueno, y Pérez-Manzano, 2018) y den respuesta de forma urgente a la situación de emergencia planetaria en la que nos encontramos. Prueba de ello son iniciativas como el Decenio de la Educación para el Desarrollo Sostenible (DEDS) 2005-2014, que ha dado paso, en 2015, al Programa de Acción Mundial de Educación para el Desarrollo Sostenible (UNESCO, 2014) y a la actual campaña E2030 Educación para Transformar vidas (UNESCO, 2015; Gil-Pérez y Vilches, 2017; Murga-Menoyo, 2018). En esta última campaña se aprobó la nueva Agenda 2030, en la que se establecen una serie de metas (los 17 Objetivos de Desarrollo Sostenible) con alusión explícita 

¿FORMA EN SOSTENIBILIDAD EL SISTEMA UNIVERSITARIO ESPAÑOL?

a la educación para la sostenibilidad y a la necesidad de reorientar los procesos formativos en todos los ámbitos de la educación (Murga-Menoyo, 2018). Concretamente, el objetivo 7 está orientado explícitamente a tales logros. En él se establece la importancia de asegurar la adquisición, por parte de los estudiantes, de teorías y prácticas que promuevan el desarrollo sostenible y la ciudadanía global.

Existe consenso académico en situar a la universidad como el ámbito más adecuado para liderar la creación de escenarios y formas de educación para la sostenibilidad (Martínez, 2018; Pérez-Franco, et al., 2018), puesto que el alumnado que se está formando en las aulas universitarias son las personas que en el futuro ocuparán los puestos profesionales de los diferentes escenarios laborales, lo cual constituye una fuerza de cambio importante para dar respuesta a los retos a los que nos enfrentamos (Jiménez- Fontana, 2016; Gil-Pérez y Vilches, 2017; Cheang, So, Zhan \& Tsoi, 2017; AznarMinguet, Ull, Martínez-Agut, y Piñero, 2017).

En este sentido, redes europeas como Copernicus, formada en 2005 por más de 300 universidades, han planteado propuestas específicas para avanzar en la integración de la sostenibilidad en los títulos universitarios. En ese mismo año 2005, la UNESCO señaló la importancia de integrar la sostenibilidad en la formación inicial y continua del profesorado, y emitió una serie de directrices y recomendaciones destinadas a reorientar la formación del profesorado. En esta línea, existen proyectos como University Educators for Sustainable Development (US4SD), desde el que se pretende dar soporte al desarrollo de educadores universitarios para la sostenibilidad, proporcionando un valioso enfoque para definir las actividades a realizar (Antúnez, 2017).

En España, desde la década de los 90 se ha producido un aumento de interés en todo lo relacionado con la sostenibilidad en el ámbito universitario. Se han llevado a cabo diferentes iniciativas, como por ejemplo: la Red de investigación en Educación para la Sostenibilidad de Cataluña (EDUSOST); el Grupo EDUCAMDES, de la Cátedra Unesco de Educación Ambiental y Desarrollo Sostenible de la UNED (Murga-Menoyo, 2018); el grupo de Ambientalización curricular ACUVEG, de la Universitat de Valencia; y la red ACES (Red de Ambientalización Curricular de los Estudios Superiores). ACES trabaja en el diseño de modelos, criterios e instrumentos de ambientalización de los estudios superiores a través del Programa de Ambientalización Curricular de los Estudios Superiores. Diseño de Intervención y Análisis del Proceso, que fue aprobado en 2001. El modelo ACES, basado en diez indicadores del nivel de ambientalización de los currículos, se ha implantado en el plan de formación del profesorado de la Universitat de Girona (Geli, Junyent, y Arbat, 2005). 

¿FORMA EN SOSTENIBILIDAD EL SISTEMA UNIVERSITARIO ESPAÑOL?

La Conferencia de Rectores de Universidades Españolas (CRUE) aprobó en septiembre de 2002 la creación de la Comisión sectorial para la Calidad Ambiental, el Desarrollo Sostenible y la prevención de riesgos (CADEP). Esta comisión se creó con el objetivo de recoger diferentes experiencias universitarias españolas sobre la gestión ambiental, los avances en la ambientalización de la comunidad universitaria y sus trabajos en prevención de riesgos, así como para mejorar la cooperación entre las mismas (Junyent y Geli de Ciurana, 2008; Larrán y Andrades, 2015). Específicamente en el ámbito de la sostenibilidad curricular, se destaca la aprobación en 2005 de un documento titulado Directrices para la sostenibilidad curricular, remitido a todas las universidades españolas y actualizado en 2011 (Aznar-Minguet, et al., 2017; Álvarez-García, Sureda, Negre, y Comas-Forgas, 2018; CRUE, 2012).

Diversos estudios (Cheung, et al., 2018) muestran que, a pesar de los esfuerzos realizados hasta el momento, los avances han sido lentos e insuficientes. Como señalan Aznar-Minguet, et al. (2017), "la literatura y la investigación en el área hasta la fecha muestran muy pocos ejemplos de cambio curricular a gran escala y las experiencias disponibles son puntuales y escasas" (p.227). Sigue habiendo una escasa incidencia en la elaboración de planes formativos que integren de manera sistémica la sostenibilidad, y el currículo y la pedagogía siguen siendo los aspectos menos abordados en los esfuerzos para reorientar la educación superior hacia la sostenibilidad (Antúnez, 2017). Las conclusiones de algunos estudios apuntan a que, si bien el profesorado es el eje central en cualquier proceso de innovación, no se está consiguiendo la implicación de este ni la formación de estudiantes como futuros líderes de la sociedad (Solís y Valderrama, 2015; Antúnez, 2017).

Por lo tanto, se vislumbra que uno de los mayores retos de las universidades para el siglo XXI es promover y mejorar la formación, para conseguir profesionales críticos y capaces de actuar bajo los principios de la sostenibilidad. En este contexto, cobra especial importancia la nueva campaña de la UNESCO, E2030 Educación para Transformar Vidas, que plantea orientaciones pedagógicas sobre qué se debe hacer para mejorar la introducción de la educación para la sostenibilidad (Murga-Menoyo, 2018). En este informe se hace hincapié en la importancia de generar entornos de aprendizaje centrados en el estudiante; se destaca la necesidad de hacer una pedagogía transformadora y orientada a la acción, que favorezca el aprendizaje auto-regulado, la participación y la colaboración; y se propone la resolución de problemas desde un abordaje interdisciplinar y transdisciplinar, poniendo en juego distintos tipos de aprendizaje en contextos diferentes (UNESCO, 2017). 

¿FORMA EN SOSTENIBILIDAD EL SISTEMA UNIVERSITARIO ESPAÑOL?

En esta línea se sitúa el proyecto EDINSOST, Educación e innovación social para la sostenibilidad. Formación en las Universidades españolas de profesionales como agentes de cambio para afrontar los retos de la sociedad (Segalàs, et al., 2018). En EDINSOST participan 59 investigadores que han analizado 13 titulaciones de 10 universidades españolas. El objetivo general del proyecto es avanzar en la innovación educativa, con el fin de dotar a los futuros titulados de las competencias necesarias para catalizar el cambio hacia una sociedad más sostenible. Para ello, se ha elaborado un mapa de sostenibilidad de las diferentes titulaciones involucradas, a partir del cual se pueden establecer las necesidades de formación en sostenibilidad del profesorado. El proyecto tiene entre sus objetivos analizar el tipo de formación en sostenibilidad que se ofrece actualmente en las universidades, y para ello pone el foco en los estudiantes universitarios con el objetivo de comprobar las competencias en sostenibilidad que adquieren a lo largo de su formación universitaria, y analizar su percepción respecto a la formación recibida.

\section{METODOLOGÍA}

El objetivo general de este trabajo es conocer y analizar las competencias en sostenibilidad del alumnado universitario y de egresados de diferentes titulaciones, así como su percepción respecto a la formación recibida en sostenibilidad. Para conseguirlo, nos propusimos los siguientes objetivos específicos:

- conocer la participación e implicación del alumnado en materia de sostenibilidad;

- indagar sobre los conocimientos en sostenibilidad del alumnado;

- examinar la percepción del alumnado sobre sus competencias en sostenibilidad;

- explorar el proceso de aprendizaje de la sostenibilidad en la universidad.

Para alcanzar estos objetivos, hemos realizado una investigación descriptiva-interpretativa de tipo cualitativo, empleando como técnica de recogida de información el grupo de discusión, lo que nos ha permitido conocer las opiniones del alumnado e interpretar los estados subjetivos de los participantes en el estudio. 

¿FORMA EN SOSTENIBILIDAD EL SISTEMA UNIVERSITARIO ESPAÑOL?

\section{Participantes}

La selección de la muestra se realizó en función de los siguientes criterios:

- alumnado de $4^{\circ}$ curso y/o egresado;

- existencia de equidad en cuestión de género;

- grupos formados por al menos 5 participantes.

La Tabla 1 presenta la muestra, formada por 29 participantes de 4 universidades españolas. Para seleccionar las titulaciones se usó el criterio de diversidad y oportunidad, y se escogieron las titulaciones del proyecto más relacionadas con las dimensiones de la sostenibilidad (económicaempresa, social-educación, ambiental-ingeniería).

Tabla 1

Participantes en los grupos de discusión

\begin{tabular}{|c|c|c|}
\hline Universidad & Titulación & Participantes \\
\hline $\begin{array}{l}\text { Universidad de } \\
\text { Salamanca }\end{array}$ & Grado Educación Social & 10 ( 4 chicos y 6 chicas) \\
\hline Universidad de Sevilla & $\begin{array}{l}\text { Grados Pedagogía y } \\
\text { Educación Primaria }\end{array}$ & 5 ( 3 chicas y 2 chicos $)$ \\
\hline $\begin{array}{l}\text { Universidad Politécnica } \\
\text { de Madrid }\end{array}$ & $\begin{array}{l}\text { Grados de Ingenierías } \\
\text { Informáticas }\end{array}$ & 5 ( 4 chicos y 1 chica $)$ \\
\hline \multirow[t]{2}{*}{$\begin{array}{l}\text { Universitat Internacional } \\
\text { de Catalunya }\end{array}$} & $\begin{array}{l}\text { Grado Administración y } \\
\text { Dirección de Empresas }\end{array}$ & 9 ( 6 chicas y 3 chicos $)$ \\
\hline & & $\begin{array}{l}\text { Total: } 29 \text { participantes } \\
(17 \text { chicas }-59 \%) \\
(12 \text { chicos }-41 \%)\end{array}$ \\
\hline
\end{tabular}

\section{Instrumentos de recogida y análisis de información}

El diseño del grupo de discusión fue realizado conjuntamente por las cuatro universidades participantes junto con el coordinador del proyecto. Dada la amplitud de la temática, se decidió que la recogida de información se limitaría a la estrictamente necesaria para dar respuesta a los objetivos planteados. 

¿FORMA EN SOSTENIBILIDAD EL SISTEMA UNIVERSITARIO ESPAÑOL?

La elaboración del sistema de categorías se ha llevado a cabo a través de un proceso deductivo-inductivo. Es deductivo en cuanto a que se partió de una revisión bibliográfica y conceptual del objeto de estudio, lo cual facilitó un marco teórico para definir las macrocategorías (Osses, Sánchez, e Ibáñez, 2006). Se realizaron reuniones periódicas en cada universidad con carácter presencial, y reuniones virtuales conjuntas vía Skype, para delimitar, consensuar y definir las categorías y subcategorías. Este segundo paso, junto con el posterior debate entre investigadores, permitió asegurar el cumplimiento de las condiciones que debe reunir un sistema de categorías: exclusión mutua; homogeneidad; pertinencia; objetividad y fidelidad; y productividad (Pérez-Serrano, 1994), lo que a su vez asegura que la información que se recoge en cada una de las categoría y subcategorías da respuesta a los objetivos de investigación planteados, proporcionando consistencia interna al instrumento. Se redactó un documento para definir los requisitos que debían cumplir los participantes en los grupos focales, el rol del moderador y del observador, y otros aspectos a tener en cuenta en el transcurso de la discusión (ambiente distendido, cómodo y de confianza entre los participantes). El documento también recoge las pautas para la transcripción, codificación y análisis, así como el sistema de categorías a utilizar y las preguntas a plantear en los diferentes grupos.

Una vez diseñado el sistema inicial de categorías, se llevó a cabo un grupo focal piloto en la Universidad de Sevilla y se realizó su correspondiente transcripción y análisis. El objetivo de este grupo piloto era valorar si en el discurso de los participantes emergía nueva información relevante que pudiera no estar recogida en ninguna de las categorías propuestas. Como resultado se decidió mantener la propuesta inicial, incluyendo una nueva categoría denominada "cajón de sastre" en la que se recogerían aquellas unidades textuales que no se correspondieran a ninguna de las categorías propuestas. El "cajón de sastre" permite valorar si la información recogida que no pertenece a ninguna categoría es de suficiente importancia como para requerir la incorporación de nuevas categorías. El "cajón de sastre" se mantuvo para el resto de grupos focales, lo que ha permitido incluir información emergente del discurso, cumpliendo así con el carácter inductivo.

El procedimiento descrito nos permitió confirmar la validez y fiabilidad de las categorías, ya que la categorización y codificación de la prueba piloto fue realizada por tres investigadores de forma independientes con el objetivo de valorar el grado de acuerdo entre codificadores. Este proceso se llevó a cabo en base a unos criterios y normas de categorización y codificación establecidos y consensuados a priori por el equipo investigador, lo cual facilitó el proceso y la toma de decisiones. Posteriormente, se realizó una reunión entre los codificadores y el resto de miembros del 

¿FORMA EN SOSTENIBILIDAD EL SISTEMA UNIVERSITARIO ESPAÑOL?

equipo investigador para reflexionar respecto a las decisiones adoptadas por los codificadores y valorar posibles modificaciones en el sistema de categorías. Finalmente, se realizó una consulta a tres expertos externos a la investigación para asegurar la validez del sistema de categorías propuesto, dando como resultado el sistema de categorías definitivo.

El diseño definitivo del instrumento que se usó en los grupos de discusión se presenta en la Tabla 2.

Tabla 2

Sistema de categorías y preguntas

\begin{tabular}{ll}
\hline Categoría & Preguntas \\
\hline $\begin{array}{l}\text { Concepción } \\
\text { Sostenibilidad }\end{array}$ & $¿$ ¿ué es para vosotros la sostenibilidad? \\
\hline
\end{tabular}

Importancia ¿Pensáis que es importante incorporar la

Sostenibilidad sostenibilidad en los estudios universitarios? ¿Por qué?

Participación ¿Qué programas o proyectos conocéis que fomenten la participación y el compromiso en la mejora socioambiental? ¿Habéis participado?

Conocimientos ¿Teníais conocimientos de sostenibilidad antes de previos la realización de vuestros estudios universitarios? ¿Cuáles?

Preparación en ¿Consideráis que la carrera os está preparando/os ha la Universidad preparado para trabajar la sostenibilidad en vuestra profesión? ¿Por qué?

Asignaturas ¿Qué asignatura consideras que ha incorporado más la sostenibilidad en tu paso por la universidad?

Metodologías y ¿Qué metodología se empleaba en la asignatura que recursos consideras que ha incorporado más la sostenibilidad en tu paso por la universidad? ¿Qué procedimientos y recursos conocéis para introducir la perspectiva de la sostenibilidad en vuestra práctica?

Competencias ¿Cuál es vuestra visión respecto a integrar el de enfoque de la sostenibilidad en vuestros proyectos sostenibilidad profesionales? ¿Pensáis que tenéis las competencias necesarias? ¿Cuáles serían esas competencias?

Roles, Relaciones y favorecer la adquisición de competencias para la clima del aula sostenibilidad? 


\begin{tabular}{ll}
\hline Categoría & Preguntas \\
\hline Evaluación & $\begin{array}{l}\text { ¿Cómo consideráis que se pueden evaluar las } \\
\text { competencias en sostenibilidad? ¿Cómo se puede } \\
\text { demostrar que se han adquirido? }\end{array}$ \\
\hline $\begin{array}{l}\text { Organización } \\
\text { curricular }\end{array}$ & $\begin{array}{l}\text { ¿Qué posibles obstáculos/facilidades encuentran los } \\
\text { profesores para organizar estrategias de enseñanza } \\
\text { activa (ApS, ABP, etc.), derivados de la organización } \\
\text { disciplinar de los planes de estudios? Propuestas de } \\
\text { mejora }\end{array}$ \\
\hline $\begin{array}{l}\text { Motivación y } \\
\text { compromiso del } \\
\text { profesorado }\end{array}$ & $\begin{array}{l}\text { ¿Consideráis que el profesorado está motivado y } \\
\text { mantiene compromiso con la sostenibilidad? ¿Por qué? }\end{array}$ \\
\hline
\end{tabular}

\section{Procedimiento}

Para la realización de los diferentes grupos de discusión, seleccionamos dos personas en cada universidad con los siguientes roles:

- moderador: responsable de dinamizar la discusión, formular las preguntas y dinamizar la fluidez de las intervenciones, procurando intervenir lo mínimo posible para garantizar que la información emitida no influyera en las opiniones y respuestas de los participantes.

- observador: encargado de anotar posibles aspectos que el audio pudiera no recoger. No podía intervenir en la discusión.

El lugar para realizar los grupos de discusión debía facilitar la comunicación entre todos los participantes. La duración de los grupos de discusión fue aproximadamente de 90 minutos, y finalizaron cuando se consideró que toda la información que se necesitaba había sido recogida. Todos los grupos fueron grabados en audio y transcritos para su posterior codificación y categorización. Para el análisis de los datos, se utilizó el método tradicional (Fernández-Núñez, 2006).

\section{RESULTADOS}

Esta sección presenta las ideas y aportaciones más relevantes que permiten dar respuesta a los objetivos planteados. Las ideas y aportaciones se han agrupado en función de las categorías definidas en el diseño previo, 

¿FORMA EN SOSTENIBILIDAD EL SISTEMA UNIVERSITARIO ESPAÑOL?

señaladas en el texto en negrita. En las citas textuales utilizadas en esta sección se incluye, entre paréntesis, la universidad en la que se recogió la intervención.

En todos los grupos se mencionó la importancia de la sostenibilidad. Hubo un acuerdo generalizado en considerar que se debe incluir formación en sostenibilidad en todas las áreas y en todos los niveles educativos, no solo en la universidad: "ya no pertinente, sino estrictamente necesario, dar un mínimo de formación. Lo ideal es que, independientemente del escalafón en el que hayas estudiado, sepas de qué va el tema" (UPM). Los participantes también consideran que "la sociedad cada vez es más consciente de estas cosas" (UIC), valorando la necesidad de la implicación ciudadana y la presión social, en particular hacia las empresas: "ahora mismo ya es una presión social, como empresa tienes que dar la imagen de que eres sostenible, si no, no te compran" (UIC). No obstante, aunque los participantes conocen el concepto de sostenibilidad, no saben definirlo. Fundamentalmente, lo asocian con el reciclaje y con el equilibrio entre producir-consumir, pero no tienen una visión holística del mismo: "no sé bien qué es la sostenibilidad, sé lo que es el reciclaje y el cuidado del medio ambiente, ¿pero sostenibilidad?" (US).

En cuanto a sus experiencias de participación en proyectos, programas, acciones sostenibles, etc., hacen alusión a hábitos y acciones cotidianas, unas veces en el ámbito familiar y otras en referencia a iniciativas ciudadanas sobre reciclaje, uso de energías renovables, donación de alimentos o ropa, campañas para el fomento del uso del transporte público, etc.: "en mi pueblo hace como un año que se ha empezado a hacer el puerta a puerta, a reciclar de forma más consciente, y ahora casi el 100\% del pueblo recicla" (UIC). Además, se hace referencia a la conciencia ecológica que hay detrás de esas acciones: "yo creo que mi madre me lo decía por la factura, por el ahorro, igual que lo de apagar la luz cuando salieras de la habitación. Pero hoy nosotros, con la formación que tenemos y también por la sociedad, que ha avanzado, podemos ser capaces de interpretar que eso es conciencia ecológica, porque no es bueno gastar sin sentido" (USAL). También se mencionan algunas iniciativas de componente técnico y cercano a su perfil profesional: "como becario en la Cátedra Accenture-UPM de Big Data, con el análisis de datos de calidad del aire proporcionados por el Ayuntamiento de Madrid" (UPM).

En cuanto a sus conocimientos sobre sostenibilidad, los estudiantes de las cuatro universidades coinciden en señalar que tienen escasa formación: "es que salimos de la universidad sin saber cosas básicas. (...) Nos estamos cargando el planeta y parece que no nos damos cuenta, porque realmente nunca se nos ha hecho demasiado hincapié" (US). Aseguran que la formación recibida antes de la universidad se centraba fundamentalmente en temas de sostenibilidad ambiental como el reciclaje y el ahorro energético, tanto en 

¿FORMA EN SOSTENIBILIDAD EL SISTEMA UNIVERSITARIO ESPAÑOL?

la escuela como en el ambiente familiar, pero sin fomentar una conciencia de sostenibilidad clara. Aluden, fundamentalmente, a la materia Educación para la Ciudadanía: "en el colegio disponían de una optativa de ciudadanía donde se reflexionaba sobre sostenibilidad" (UPM). No obstante, algunos estudiantes se muestran críticos en el análisis de los conocimientos previos y las carencias en la formación recibida: “¿qué se enseña por sostenibilidad en la escuela? Por ejemplo, los tres contenedores. Y para de contar, Y en los tres contenedores hay un interés de dinero por detrás. Porque el reciclaje son empresas nada más" (US).

Tampoco la preparación y formación recibida en la Universidad salen bien paradas. Los estudiantes las consideran necesarias e imprescindibles, pero destacan que la sostenibilidad apenas se aborda a lo largo de la carrera, y no se sienten preparados para incorporar esta competencia en su carrera profesional: "si quiero incluir eso en mi proyecto profesional, necesito formación” (US). En esta línea se menciona que, en ocasiones, la formación depende de su implicación personal en la búsqueda de información y de formación complementaria: "son temas en los que yo me informo o me entero, por mí misma, quizás por internet o por las noticias... pero no creo que la universidad me esté dando los medios suficientes para conocer esos temas y poder trabajarlos con los niños" (US).

En alguno de los grupos se identifican otras carencias, como por ejemplo el poco tiempo dedicado al desarrollo de competencias transversales, y que no se fomentan actividades extracurriculares dedicadas a estas cuestiones o prácticas con repercusión positiva en su formación en sostenibilidad: "las competencias transversales, sí, se ponen en la guía y voy a tratar de justificar que te lo he enseñado, pero no le he dedicado tiempo, y eso son carencias" (UPM). Sin embargo, consideran que hay veces en que el profesorado no hace alusión a la sostenibilidad como concepto, pero trabaja cuestiones éticas y sociales que ellos sí identifican en esa línea: "en estos temas, no solo del reciclado, sino también de la convivencia, la prevención de la violencia y algunas otras cosas, que eso también es sostenibilidad social ¿no?, pues también nos preparan, aunque no lo llamen sostenibilidad" (USAL).

Respecto a las asignaturas concretas en donde han recibido formación, mencionan planes de estudio como el Grado en Pedagogía de la US o el Grado de Educación Social en la USAL, que tienen materias optativas vinculadas a la sostenibilidad: "en la asignatura [Educación Socioambiental] sí que hemos visto lo que es la sostenibilidad, las definiciones y eso" (USAL); "la asignatura de Educación Ambiental, antes era obligatoria y ahora es optativa, y eso en Pedagogía, porque en primaria creo que ni siquiera tienen Educación Ambiental, la tienen en infantil" (US). Los grados de ingenierías informáticas de la UPM tienen una asignatura obligatoria, Aspectos Éticos 

¿FORMA EN SOSTENIBILIDAD EL SISTEMA UNIVERSITARIO ESPAÑOL?

y Sociales, donde se trabaja la sostenibilidad, y en el Trabajo Final de Grado (en adelante TFG) se les pide una reflexión sobre aspectos éticos, sociales, legales y ambientales relacionados con el proyecto. Sin embargo, los estudiantes manifiestan no saber cómo enfrentarse a ello por falta de formación o porque la temática no es apropiada: "hay TFG que no tienen una aplicación real" (UPM). Respecto al resto de materias que componen los planes de estudio, los alumnos consideran que no se detienen en la formación en sostenibilidad.

Se presentan a continuación las opiniones de los participantes sobre cómo consideran que debería ser la formación universitaria en sostenibilidad.

En cuanto a las competencias que consideran más importantes, destacan la importancia de tomar conciencia y la importancia de la reflexión: "si no nos creemos lo que está pasando, no podemos transmitirlo" (US). Consideran que el clima del aula debería ser un espacio abierto a la reflexión: "que sea un lugar abierto a debate y en el que todos puedan aprender de las opiniones de los otros" (UPM). Pero los estudiantes son conscientes de que eso no es suficiente, y es necesario tener estas competencias para llevar a la práctica la sostenibilidad y aplicar lo aprendido en el ámbito profesional: "Si creamos la conciencia, pero luego no decimos las acciones a tomar para cambiarlo... Sí, creamos la conciencia, decimos "esto está mal", pero ¿y qué?, ¿qué hacemos?, ¿no tomamos ninguna medida? Sabemos que está mal y lo seguimos haciendo" (US); "la asignatura podría poner en práctica sus contenidos con ejemplos prácticos, a la vez que el alumno gana cultura en materias de sostenibilidad y otros aspectos de responsabilidad social" (UPM).

Las metodologías docentes que los estudiantes consideran más apropiadas son las que relacionan el trabajo de las competencias de sostenibilidad con situaciones reales, tanto dentro como fuera del aula: "nos han presentado casos de escándalos de empresas" (UIC); "si hicieran más proyectos como los que hicimos en ambiental con los huertos, por ejemplo, donde ves la realidad" (USAL); "que vengan personas a contarte cosas que pasan en el ambiente laboral" (UPM).

También se habla de la posibilidad de involucrar al alumnado en proyectos que tengan un impacto social o ambiental, destacando el aprendizaje-servicio como una herramienta de gran utilidad: "para mí lo mejor [es] el aprendizaje servicio, porque nos permite a nosotros aprender las cosas que vemos en las asignaturas (...), y luego pues vamos alli y les enseñamos, les ayudamos, eso es lo mejor" (USAL); "yo creo que la universidad debería de fomentar y financiar este tipo de proyectos [colaborativos] y que los alumnos formen parte activa de dichos proyectos" (UPM). Se comenta que, si 
ROCÍO VALDERRAMA-HERNÁNDEZ, LUCÍA ALCÁNTARA RUBIO, FERMÍN SÁNCHEZ-CARRACEDO, DAVID CABALLERO, SARA SERRATE, DOLORS GIL-DOMÉNECH, SALVADOR VIDAL-RAMÉNTOL, RAFAEL MIÑANO ¿FORMA EN SOSTENIBILIDAD EL SISTEMA UNIVERSITARIO ESPAÑOL?

se pretende implicar a los estudiantes en proyectos de aprendizaje-servicio, es importante poner en valor el aprendizaje de competencias relacionadas con la titulación que comporten estas experiencias: "te ayudan a nivel personal, sobre todo de trabajo en equipo y de empatía, de ponerte en lugar del otro, aprender a pensar de forma diferente a la tuya, que la persona de al lado puede tener ideas mejores que las tuyas; hay que decir que te va a servir para tu futuro y tu futuro profesional" (UPM).

Otras metodologías y recursos que se sugieren a la hora de trabajar la sostenibilidad, más relacionadas con el desarrollo de la reflexión crítica y la sensibilización, son el uso de diarios como recursos de aprendizaje, el análisis de noticias actuales o la realización de trabajos y exposiciones: "nosotros, haciendo el diario reflexionamos mucho porque metemos un montón de experiencias" (US); "que un alumno que tenga un tema que le interese, como puede ser el medio ambiente, venga y lo explique en clase y de esa manera conciencie a sus compañeros" (US).

Algunos estudiantes dan importancia a que se potencie la motivación y se involucre al alumnado, y que el aprendizaje sea significativo y permanezca a medio y largo plazo: "por parte del alumnado, el principal problema es el desinterés por determinadas asignaturas o contenidos; (...) se debe trabajar en su motivación, y en su inclusión en estas iniciativas" (UPM).

La evaluación de las competencias en sostenibilidad se considera, en general, algo necesario y positivo, pero complicada de llevar a la práctica: "me parece muy difícil evaluar ese proceso" (US); "algunas cosas se ven a largo plazo y no se pueden evaluar en el momento" (USAL). Consideran importante que la evaluación sea más cualitativa que cuantitativa, que vaya más allá de los conocimientos teóricos y que contemple aspectos como las habilidades de reflexión o los cambios en comportamientos cotidianos. Consideran algunas técnicas metodológicas mencionadas anteriormente como instrumentos de evaluación adecuados: "trabajos que verdaderamente inviten al alumno a reflexionar" (UPM); "[en relación al diario] se ve esa mejoría durante el cuatrimestre, porque al final vamos elaborando nuestro día a día trabajando más cosas, buscando información no sé dónde, y entonces esa sostenibilidad se ve en el proceso desde el primer día de clase hasta el día de la entrevista" (US). No obstante, algún comentario previene del riesgo de que la evaluación se convierta en un trámite y se olvide que es fundamental la motivación del alumno: "Sirve en cuanto a conseguir que los profesores las incluyan en el temario, pero es habitual que no se refleje en beneficios para el alumno, sino en un trámite como la realización de un trabajo escrito o la resolución de una pregunta de un tipo determinado en el examen" (UPM). 

¿FORMA EN SOSTENIBILIDAD EL SISTEMA UNIVERSITARIO ESPAÑOL?

En tres de los grupos se destaca la importancia del rol del profesorado, así como de su compromiso y motivación. En la UPM se hace referencia al papel del profesorado en la docencia y también en sus otras actividades, sugiriendo que el profesorado debería transmitir en la docencia lo que hace en sus proyectos y las implicaciones de los mismos: "yo estoy seguro que los profesores de esta escuela están involucrados en proyectos y que esos proyectos tienen una repercusión. Cuando un alumno entra, los referentes son tus profesores. Qué mejor ejemplo que ellos te cuenten lo que están haciendo y cómo impacta. Que lo cuenten en las clases, o se pueden crear espacios abiertos en otros ámbitos" (UPM). Además, consideran fundamental la orientación y la experiencia del tutor si se trabajan aspectos de sostenibilidad en el TFG. Por el contrario, da la impresión de que algunos estudiantes de la USAL o la US piensan que sus profesores no tienen competencias en sostenibilidad: " $e l$ problema es si el profesorado está preparado para eso porque, claro, cómo nos van a formar si a lo mejor ellos no saben lo que es la sostenibilidad" (USAL), y se quejan de que la mayoría de profesores no habla nunca de sostenibilidad $\mathrm{y}$, en todo caso, dejan que sea el propio alumno el que aprenda los conceptos por sí mismo: "quitando con [los profesores de la materia de sostenibilidad], no hemos visto nada directamente que nos hayan dicho los profesores: esto es sostenibilidad, o esto es para tener un desarrollo más sostenible y tal" (USAL); También se destaca la importancia de "cómo" se transmite: "que el profesor nos haga sentir partícipes. De ese modo te involucras más" (US).

La última categoría definida es la relativa a la organización curricular, considerando los participantes que los temas de sostenibilidad deberían estar incluidos en el currículo en asignaturas obligatorias: "para que se dé la importancia que realmente se merece hay que incorporarlo en el currículo, porque parece que si no aparece en el currículo es que no hay que ni hablarlo" (US); "me parece especialmente importante que se trabaje en más asignaturas, o bien que en vez de optativa la asignatura sea obligatoria y que se dé antes, no en el último cuatrimestre de cuarto" (USAL). Sobre cómo puede integrarse la sostenibilidad en el currículo, los estudiantes se muestran de acuerdo en que se debería introducir en todas las asignaturas de forma trasversal, pero en el grupo de la UIC echan en falta "una asignatura de sostenibilidad que integrase las distintas aplicaciones en el ámbito económico y empresarial. Así, de algún modo recogería todo lo trabajado en las distintas asignaturas" (UIC). En la UPM, donde sí existe ese tipo de asignatura, se cuestiona el impacto que pueda tener si está en primer curso: "lo que se trata es de transmitir esos valores que tienes que tener interiorizados como profesional, y un alumno de primero no se siente así, y quizás es demasiado pronto para llegar a la reflexión de si hago esto pasa esto. Está más centrado en aprender cómo hacerlo, y luego ya se podrá parar a reflexionar si está bien lo que hago o debería hacerlo de otro modo" (UPM). 

¿FORMA EN SOSTENIBILIDAD EL SISTEMA UNIVERSITARIO ESPAÑOL?

Varios grupos consideran apropiado que las competencias en sostenibilidad estén en el TFG, bien a la hora de proponer un plan de negocio y proponer alternativas más sostenibles, o bien considerando las implicaciones o impactos que pueda tener el proyecto desarrollado. Y para que en el TFG ya se sepa cómo integrar criterios de sostenibilidad, consideran necesario que estos aspectos deben trabajarse previamente en otras asignaturas: "en gestión de proyectos se podría poner algo extra, igual que en el TFG. O en Verificación y Validación, se podría poner que en un apartado de esa práctica se vean sus implicaciones. La gente puede pensar 'qué rollo', pero es algo que hay que asumir y tiene que venir ligado con ello" (UPM).

Finalmente, nos parece importante reseñar que en los grupos también se recogen algunos comentarios sobre aspectos interesantes que no se enmarcan en las categorías definidas por no estar directamente relacionados con la actividad universitaria, pero que son reflejo de las inquietudes de los estudiantes. En general, están relacionados con la asimilación de la sostenibilidad por la sociedad: "hace falta un cambio de legislación que fomente la sostenibilidad" (UIC); "el paro es un problema de sostenibilidad" (UPM); "hay que buscar modos alternativos sostenibles de vivir" (UIC); "llevar la sostenibilidad a la práctica en el entorno empresarial es difícil" (UIC).

\section{DISCUSIÓN}

En general, todos los grupos han destacado la importancia de la sostenibilidad y consideran necesaria e imprescindible la inclusión de formación en sostenibilidad en todas las áreas y niveles educativos. Sin embargo, los alumnos no acaban de saber definir el término sostenibilidad, y lo asocian más bien con reciclaje, mantenimiento de los recursos en el tiempo y equilibrio producción-consumo. Les falta una visión holística, lo cual es razonable dado que, por un lado, manifiestan haber recibido una formación parcial -centrada principalmente en sostenibilidad ambientaldurante su etapa pre-universitaria, y, por otro, catalogan de insuficiente la preparación que se les proporciona en la universidad. De hecho, destacan que a lo largo de la carrera no se abordan apenas aspectos que tengan que ver con la sostenibilidad, lo que les lleva a afirmar que no se sienten preparados para incorporar la sostenibilidad en su carrera profesional futura. Dando respuesta al título de este trabajo, se deduce que la universidad no está formando adecuadamente en sostenibilidad a sus estudiantes. Ante este panorama, se pone aún más de manifiesto la necesidad de formar al profesorado para que, de forma transversal, trabaje la sostenibilidad bajo distintos puntos de vista en sus asignaturas. En esta línea, los grupos de discusión han aportado información valiosa en cuanto a cómo debería ser 

¿FORMA EN SOSTENIBILIDAD EL SISTEMA UNIVERSITARIO ESPAÑOL?

la formación universitaria en relación a la sostenibilidad, lo que puede servir para establecer hojas de ruta que ayuden a cubrir las carencias detectadas.

En primer lugar, esta formación debería centrarse en el desarrollo de competencias transversales, principalmente aquellas -como el pensamiento crítico- que fomenten la reflexión y el debate. Sin embargo, no es suficiente con el "saber", sino que hace falta que el aula sea un espacio de concienciación en el que se potencie el "saber aplicar". De ello son conscientes los estudiantes, que destacan la necesidad de llevar a la práctica la sostenibilidad y poder aplicar lo aprendido en su ámbito profesional.

En segundo lugar, se apunta a metodologías docentes que pueden resultar de elevada utilidad a la hora de promover competencias en sostenibilidad. Entre otras, se hace especial alusión al aprendizaje-servicio como metodología que permite involucrar al alumnado en proyectos con impacto social o ambiental. También se apunta al uso de diarios como recursos de aprendizaje para reflexionar y sensibilizar sobre la sostenibilidad. En cuanto a la evaluación, se sugiere que sea más cualitativa, yendo más allá de los conocimientos teóricos y teniendo en cuenta aspectos como las habilidades de reflexión o los cambios en comportamientos cotidianos.

En tercer lugar, se destaca el rol del profesorado -haciendo alusión a su compromiso, motivación y al cómo transmite- a la hora de involucrar y motivar a los alumnos para que el aprendizaje sea significativo y permanente. Los estudiantes manifiestan que cuando un profesor se involucra en un proyecto, su ejemplo hace que ellos mismos valoren más las acciones que se llevan a cabo, y se sientan más motivados a desarrollarlo.

Por último, es importante apuntar a la organización curricular, ya que se considera de vital importancia el hecho de incluir la sostenibilidad en asignaturas obligatorias del currículo. En las discusiones de los distintos grupos surgieron diversas propuestas, relativas tanto al trabajo transversal en todas las asignaturas que pudieran tener alguna relación con la sostenibilidad, como a la existencia de asignaturas obligatorias que den una visión global de la relación de la sostenibilidad con el ámbito de la titulación que se está cursando, además de la inclusión de la sostenibilidad en el TFG. Una integración coherente y efectiva de la sostenibilidad en los planes de estudio requiere que se trabaje de forma coordinada. Por tanto, es necesario que haya varias asignaturas, que cursen todos los estudiantes, que aporten los conceptos básicos de sostenibilidad y consigan una visión holística de la misma, que evite la visión sesgada que puede proporcionar el hecho de centrarse solo en algunos aspectos concretos relacionados con una determinada materia. Es pertinente que esos aspectos se visibilicen en asignaturas específicas de la titulación y se puedan trabajar de forma 

¿FORMA EN SOSTENIBILIDAD EL SISTEMA UNIVERSITARIO ESPAÑOL?

transversal en las mismas, pues de este modo se refuerza la percepción de que la sostenibilidad no es algo ajeno a la actividad profesional. Con ese mismo objetivo, es fundamental que también se incluya la sostenibilidad en el TFG. Los mapas de sostenibilidad de las titulaciones pueden ser un instrumento muy útil y eficaz para este fin (Sánchez Carracedo, et al., 2018b).

Por todo lo anterior, abogamos por el aprendizaje activo como elemento clave para educar a los alumnos en sostenibilidad, porque permite involucrar a los estudiantes haciéndoles realizar actividades y reflexionar sobre lo que están haciendo, reflejar sus ideas y cómo las utilizan (Prince, 2004). Además, facilita la participación, independencia y responsabilidad de los alumnos, a la vez que cambia el papel de los educadores, convirtiéndolos en guías o facilitadores (Phillips, 2004). Gracias a este tipo de aprendizaje, los estudiantes pueden ver aumentada su motivación para aprender, retener más el conocimiento y comprender más profundamente el tema, adquiriendo actitudes positivas (Michael, 2006). El aprendizaje activo también facilita la adquisición de competencias transversales a través de la práctica y, consecuentemente, proporciona un aprendizaje vital que los estudiantes podrán aplicar a lo largo de sus carreras profesionales y personales (Star \& Hammer, 2008).

El presente trabajo no está exento de limitaciones, que principalmente provienen de la propia muestra -cuatro grupos focales, algunos de ellos con pocos (cinco) alumnos-. Sin embargo, teniendo en cuenta el abanico de disciplinas cubiertas -Educación, Ingeniería y Administración y Dirección de Empresas-, que guardan una estrecha relación con las distintas dimensiones -ambiental, social y económica- de la sostenibilidad, consideramos que las limitaciones que puedan derivarse del tamaño muestral quedan superadas por la diversidad de puntos de vista recogidos.

Por último, cabe destacar que los resultados de esta investigación -la escasa formación en sostenibilidad manifestada por los alumnos- nos tienen que llevar, como docentes, a una reflexión profunda y a tomar medidas que se plasmen en la formación de las próximas generaciones. Algunas universidades ya se sitúan en esta línea y han creado el Vicerrectorado de Sostenibilidad, que trata de impulsar la sostenibilidad a partir de distintas acciones. Esta urgencia se ha manifestado también en los grupos de discusión, que han dado una especial relevancia al hecho de transmitir la sostenibilidad para que sea toda la sociedad quien la asuma. Sin embargo, no hay que olvidar la necesidad de que las dimensiones social y ambiental vayan de la mano de la dimensión económica: las dos primeras no podrán mantenerse a largo plazo si, desde el punto de vista económico, los proyectos que integren la sostenibilidad no son rentables. Es por tanto clave tener 
en cuenta, a la hora de dar respuesta a este requisito, que una solución sostenible es aquella que considera de forma holística las tres dimensiones de la sostenibilidad.

\section{CONCLUSIONES Y TRABAJO FUTURO}

En este artículo se han presentado las opiniones de alumnos y egresados de cuatro universidades sobre el aprendizaje de la sostenibilidad recibido en la universidad. Se han analizado cinco titulaciones directamente relacionadas con las dimensiones de la sostenibilidad. El análisis se ha realizado mediante la técnica "grupos de discusión" usando un conjunto de categorías predefinido.

Los resultados obtenidos muestran que aún queda mucho trabajo por hacer y que el alumnado refleja la necesidad y la urgencia de realizar este trabajo. Esto debe de servir de motivación para transformar nuestra actividad como docentes y contribuir a que las universidades asuman su responsabilidad para afrontar, conjuntamente con otros actores sociales, los retos que plantea el logro de un desarrollo sostenible. Porque el futuro será sostenible o no será.

El análisis cualitativo presentado en este trabajo se completará en el futuro con un análisis cuantitativo en el marco del proyecto EDINSOST. Para realizar este análisis se ha elaborado un cuestionario que han respondido 3766 alumnos de las 10 universidades participantes en dicho proyecto (Sánchez Carracedo, et al., 2018a). Los datos obtenidos se están analizando actualmente y se espera publicar los resultados en los próximos meses.

\section{NOTAS}

1 Este trabajo ha sido financiado por el Ministerio de Economía y Competitividad a través del proyecto EDINSOST, EDU2015-65574-R. 


\section{REFERENCIAS BIBLIOGRÁFICAS}

Álvarez-García, O., Sureda-Negre, J., \& Comas-Forgas, R. (2018). Assessing Environmental Competencies of primary education pre-service teachers in Spain: a comparative study between two universities. International Journal of Sustainability, 19(1), 15-31. https:// doi.org/10.1108/IJSHE-12-2016-0227.

Antúnez, M. (2017). Problemática del proceso de sostenibilización curricular en el contexto universitario español: la formación del profesorado como catalizador [Tesis doctoral]. Universidad de Córdoba. Recuperado de https://bit.ly/2M1w0Ui

Aznar-Minguet, P., Ull, M.A., MartínezAgut, M.P., y Piñero, A. (2017). Evaluar para transformar: evaluación de la docencia universitaria bajo el prisma de la sostenibilidad. Enseñanza de las Ciencias, 35(1), 5-27.

Cheang, C., So, W., W.-M., Zhan, Y., \& Tsoi, K. (2017). Education for Sustainability using a campus ecogarden as a learning environment. International Journal of Sustainability in Higher Education, 18(2), 242-262. https://doi.org/10.1108/IJSHE-102015-0174

Cheung, T.Y., Chow, C., \& So, W. (2018). A Train-the-Trainer Design for Green Ambassadors in an Environmental Education Programme on Plastic Waste Recycling. International Research in Geographical and Environmental Education, 27(1), 2442. Doi: https://doi.org/10.1080/10382 046.2017.1285138.

Cheung, T.Y., Fok, L., Cheang, C., Yeung, C., So, W., y Chow, C. (2018). Reciclaje de plásticos en salas universitarias: un estudio de intervención mixta. Revista Internacional de Sostenibilidad en Educación Superior, 19(6), 10381052. https://doi.org/10.1108/IJSHE10-2017-0175.

CRUE (2012). Directrices para la introducción de la Sostenibilidad en el Curriculum. Recuperado de https:// bit.ly/2LZFtv1

Fernández-Núñez, L. (2006). ¿Cómo analizar datos cualitativos? Bulletí La Recerca 7. Recuperado de https://bit. ly/29CPQE4

Geli, A., Junyent, M., y Arbat, E. (2005). La Sostenibilidad en la Formación Inicial del Profesorado: Aplicación del Modelo ACES. Enseñanza de las Ciencias. Número Extra. VII (s/p). Recuperado de https://bit.ly/2Y2xKi6

Gil Pérez, D. y Vilches, A. (2017). Educación para la sostenibilidad y educación en derechos humanos: dos campos que deben vincularse. Teoría de la Educación. Revista Interuniversitaria, 29(1), 79100. http://dx.doi.org/10.14201/ teoredu29179100.

Jimenez-Fontana, R. (2016). La Evaluación en la Educación para la Sostenibilidad desde el Paradigma de la Complejidad [Tesis doctoral]. Universidad de Cádiz. Recuperado de: https://bit.ly/2Y1Tefc

Junyent, M. \& Geli de Ciurana, A.M. (2008). Education for Sustainability in university studies: a model for reorienting the curriculum. British educational research journal, 34(6), 763-782. 10.1080/01411920802041343

Larrán, M. y Andrades, F.J. (2015) Análisis de la responsabilidad social universitaria desde diferentes enfoques teóricos. Revista Iberoamericana de Educación Superior, 6, 91-107. 
ROCÍO VALDERRAMA-HERNÁNDEZ, LUCÍA ALCÁNTARA RUBIO, FERMÍN SÁNCHEZ-CARRACEDO, DAVID

CABALLERO, SARA SERRATE, DOLORS GIL-DOMÉNECH, SALVADOR VIDAL-RAMÉNTOL, RAFAEL MIÑANO ¿FORMA EN SOSTENIBILIDAD EL SISTEMA UNIVERSITARIO ESPAÑOL?

http://dx.doi.org/10.1016/S20072872(15)30005-6

Martínez, M. (2018). La enseñanza de la Justicia Ambiental en el Marco de la Educación para el Desarrollo Sostenible en la Universidad. Revista Internacional de Educación para la Justicia Social, 7(1). http://dx.doi. org/10.15366/riejs2018.7.1.003

Michael, J. (2006). Where's the evidence that active learning works? Advances in Physiology Education, 30(4), 159167. 10.1152/advan.00053.2006.

Murga-Menoyo, M. (2018). La Formación de la Ciudadanía en el Marco de la Agenda 2030 y la Justicia Ambiental. Revista Internacional de Educación para la Justicia Social, 7(1), 37-52. http://dx.doi.org/10.15366/ riejs2018.7.1.002

Osses Bustingorry, S., Sánchez Tapia, F., \& Ibáñez Mansilla, M. (2006). Qualitative research in education: towards the generation of theory through the analytical process. Estudios Pedagógicos, XXXII(1), 119133.

Pérez Franco, D., de Pro Bueno, A., y Pérez Manzano, A. (2018). Actitudes ambientales al final de la ESO. Un estudio diagnóstico con alumnos de Secundaria de la Región de Murcia. Revista Eureka Sobre Enseñanza Y Divulgación De Las Ciencias, 15(3), 3501. http://dx.doi.org/10.25267/Rev_ Eureka_ensen_divulg_cienc.2018.v15. i3.3501

Pérez-Serrano, G. (1994). Investigación cualitativa. Retos e interrogantes. II Técnicas y análisis de datos. Madrid: La Muralla.

Phillips, J.M. (2005). Strategies for active learning in online continuing education. Journal of Continuing Education in Nursing, 36(2), 77-83.
Prince, M. (2004). Does active learning work? A review of the research. Journal of Engineering Education, 93(3), 223-231. https:// doi.org/10.1002/j.2168-9830.2004. tb00809.x.

Sánchez Carracedo, F., Álvarez, M.J., Barrón, A., Caballero, López, E., Muñoz, J.M., ... y Vidal, S. (2018a). Elaboración de un cuestionario para evaluar el nivel de sostenibilidad de los estudiantes de grados en ingeniería TIC. JENUI 2018. Actas de las Jenui, 3,141-148. Barcelona.

Sánchez Carracedo, F., Segalàs, J., Vidal, E., Martín, C., Climent, J., López, D., \& Cabré, J. (2018b). Improving engineering educators' sustainability competencies by using competency maps: The EDINSOST project. International Journal of Engineering Education, 34(5), 1527-1537.

Segalàs, J., Sánchez Carracedo, F., Hernández, A., Busquets, P., Tejedor, G., \& Horta, R. (2018). The EDINSOST Project. Training sustainability change agents in Spanish and Catalan engineering Education. Proceedings of the Engineering Education for Sustainable Development Conference 2018, EESD 2018, Glasboro, New Jersey, USA, 109-116.

Solís-Espallargas, C. y ValderramaHernández, R. (2015). La educación para la sostenibilidad en la formación de profesorado. ¿Qué estamos haciendo? Foro de Educación, 13(19), 165-192. http://dx.doi.org/10.14516/ fde.2015.013.019.008

Star, C. \& Hammer, S. (2008). Teaching generic skills: Eroding the higher purpose of universities, or an opportunity for renewal? Oxford Review of Education, 34(2), 237-251. https:// doi.org/10.1080/03054980701672232. 

CABALLERO, SARA SERRATE, DOLORS GIL-DOMÉNECH, SALVADOR VIDAL-RAMÉNTOL, RAFAEL MIÑANO ¿FORMA EN SOSTENIBILIDAD EL SISTEMA UNIVERSITARIO ESPAÑOL?

UNESCO (2015). Replantear la Educación. ¿Hacia un bien común mundial? París, Francia: Unesco.

UNESCO (2014) Declaración de AichiNagoya sobre la Educación para el Desarrollo Sostenible. Paris, Francia:
UNESCO. Recuperado de https://bit. $\mathrm{ly} / 2 \mathrm{KsPgKa}$

UNESCO (2017). Educación para los Objetivos de Desarrollo Sostenible. Objetivos de aprendizaje. París, Francia: UNESCO. Recuperado de https://bit.ly/2mxGzTD 

¿FORMA EN SOSTENIBILIDAD EL SISTEMA UNIVERSITARIO ESPAÑOL?

\section{PERFIL ACADÉMICO Y PROFESIONAL DE LOS AUTORES}

Rocío Valderrama Hernández. Doctora en Pedagogía (2012) por la Universidad de Sevilla. Profesora del Departamento de Teoría e Historia de la Educación y Pedagogía Social de la Universidad de Sevilla. Su área de investigación se desarrolla en torno a la educación ambiental, sociedades inclusivas y educación, infancia, participación y desarrollo humano. Miembro del grupo de Investigación HUM 596, Educación de Personas Adultas y Desarrollo.

Lucía Alcántara. Graduada en Pedagogía (2013) por la US. Ha sido colaboradora honoraria del dpto. de Teoría e $\mathrm{H}^{\mathrm{a}}$ de la Educación en los años consecutivos hasta 2017. Sus áreas de investigación son la formación docente, la Educación para la sostenibilidad y didácticas curriculares. Desde mayo de 2018 se encuentra como investigadora en el INDESS (Instituto Universitario de Desarrollo Social y Sostenible) de la UCA; actualmente está finalizando su tesis doctoral centrada en la ambientalización curricular.

Fermín Sánchez-Carracedo. Licenciado en Informática desde 1987 y Doctor en Informática desde 1996, es profesor Titular de Universidad en el Departamento de Arquitectura de Computadores de la UPC. Sus áreas de investigación son la innovación docente y la educación para la sostenibilidad. Ha sido consultor de la UOC (1997-2010) y Vicedecano de innovación de la Facultat d'Informàtica de Barcelona-FIB (2007 -2013). Desde julio de 2013 ocupa el cargo de Adjunto de Innovación en la FIB.

David Caballero Franco. Diplomado en Educación Social, Licenciado en Pedagogía, Profesor Asociado en el Departamento de Teoría e Historia de la Educación. Universidad de Salamanca. Su Área de investigación es la Pedagogía Social y sus líneas temáticas son Educación Familiar y Desarrollo Comunitario, Pedagogía Familiar y Educación Socioambiental. Actualmente forma parte del Grupo de Investigación de Excelencia de la Junta de Castilla y León. GR209: Procesos, espacios y prácticas educativas.

Dolors Gil-Doménech. Doctora en Estudios Empresariales (2013) y licenciada con premio extraordinario en Administración y Dirección de Empresas (2005) y con primer premio nacional en Ciencias Actuariales y Financieras (2006) por la UB. Es profesora en UIC Barcelona desde 2010. Su principal línea de investigación se sitúa en el ámbito de economía de la educación, y más concretamente en la innovación docente y desarrollo competencial, si bien también investiga en los campos de la transferencia universidad-empresa y el desarrollo del espíritu emprendedor. 

¿FORMA EN SOSTENIBILIDAD EL SISTEMA UNIVERSITARIO ESPAÑOL?

Rafael Miñano. Licenciado en CC Matemáticas desde 1986 y actualmente realizando su tesis doctoral el programa de Organización Industrial de la UPM. Es profesor Titular de Escuela Universitaria en el Departamento de Matemática Aplicada a las Tecnologías de la Información y las Comunicaciones de la UPM. Sus áreas de investigación son la innovación docente y la educación para la sostenibilidad, la responsabilidad social y la ética profesional.

Sara Serrate González. Doctora en Educación por la Universidad de Salamanca (2014), Graduada en Educación Social y Licenciada en Psicopedagogía. Profesora Ayudante Doctora en la Facultad de Ciencias Sociales en el departamento de Teoría e Historia de la Educación. Es miembro del Grupo de Investigación Reconocido de la Universidad de Salamanca "Procesos, espacios y prácticas educativas". Sus líneas de investigación se centran fundamentalmente en la Pedagogía Social y la Intervención Socioeducativa.

Salvador Vidal-Raméntol. Profesor Agregado de la Facultad de Educación UIC Barcelona. Profesor de Didáctica de las Matemáticas. Doctor en Ciencias de la Educación UB (2001). Licenciado en C. Químicas, UB. Vicedecano de la F. Educación. Miembro del grupo de investigación SEI. Sus líneas de investigación giran en torno a la didáctica de las matemáticas y consumo sostenible. Director de tres tesis doctorales. Tiene distintas publicaciones, tanto en libros como artículos científicos. Evaluación muy favorable en docencia universitaria.

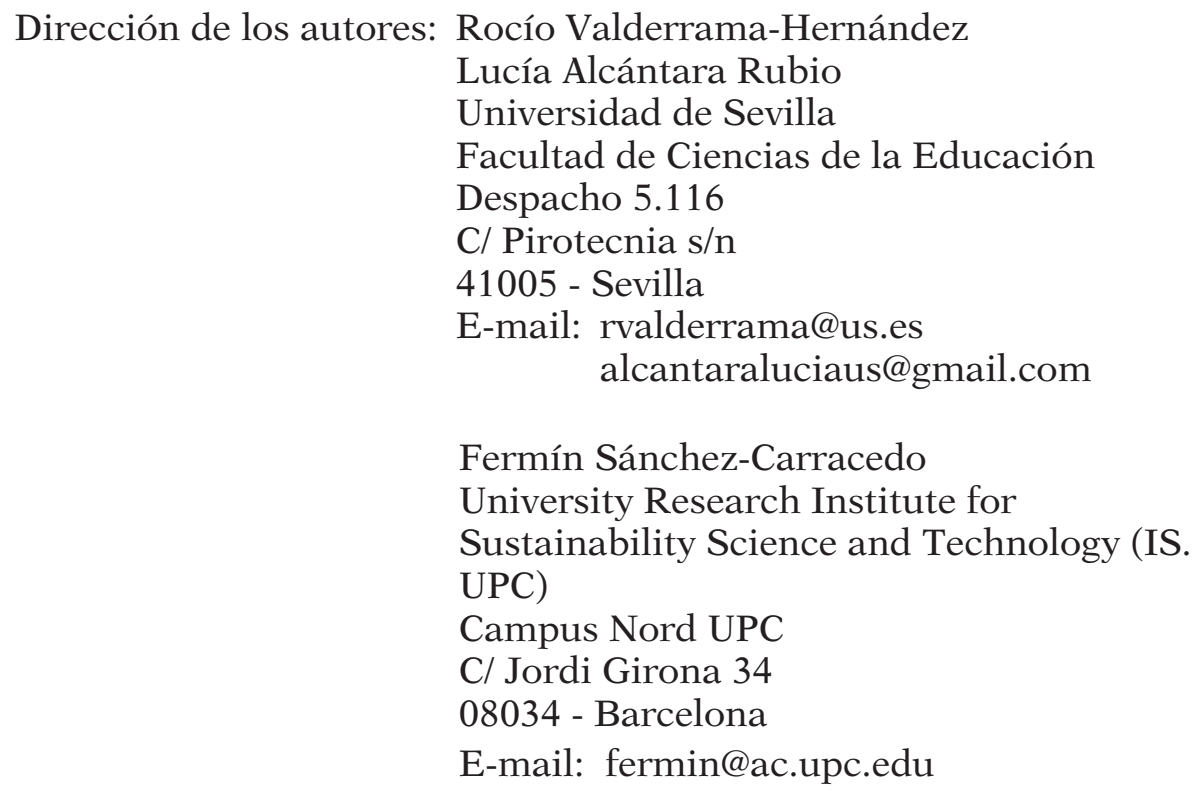


David Caballero Franco

Paseo de Canalejas, 169

Campus. Facultad de Educación

E-mail: caballero@usal.es

Dolors Gil-Doménech

Department of Economy and Business

Organisation

Universitat Internacional de Catalunya

C/ Immaculada, 22

08017 - Barcelona

E-mail: mdgil@uic.es

Rafael Miñano

Dpto. Matemática Aplicada a las TIC

ETSI Sistemas Informáticos

Campus Sur UPM

$\mathrm{C} /$ Alan Turing s/n

28031 - Madrid

E-mail: rafael.minano@upm.es

Sara Serrate González

Facultad de Ciencias Sociales

Edificio FES

Avd. Francisco Tomás y Valiente, s/n

37007 - Salamanca

E-mail: sarasg@usal.es

Salvador Vidal Raméntol

Facultad de Educación Universitat

Internacional de Catalunya

C/ Terré 11-19

08017 Barcelona

E-mail: svidal@uic.es

Fecha Recepción del Artículo: 9. Enero. 2019

Fecha Modificación del Artículo: 9. Mayo. 2019

Fecha Aceptación del Artículo: 7. Junio. 2019

Fecha Revisión para Publicación: 28. Junio. 2019 
